\title{
Replicating retroviral vectors for oncolytic virotherapy of experimental hepatocellular carcinoma
}

\author{
YIN-CHE LU ${ }^{1 *}$, YING-JU CHEN ${ }^{2 *}$, YUAN-REN YU ${ }^{2}$, YI-HUI LAI ${ }^{2}$, \\ JEN-CHIEH CHENG ${ }^{2}$, YU-FEN LI ${ }^{3}$, CHENG-HUANG SHEN ${ }^{2,4}$ and CHIEN-KUO TAI ${ }^{2}$
}

${ }^{1}$ Division of Hematology-Oncology, Ditmanson Medical Foundation Chia-Yi Christian Hospital, Chia-Yi 60002; ${ }^{2}$ Department of Life Science and Institutes of Molecular Biology and Biomedical Science, National Chung Cheng University, MinHsiung, Chia-Yi 62102; ${ }^{3}$ Institute of Biostatistics, China Medical University, Taichung 40402; ${ }^{4}$ Departments of Urology and Medical Research, Ditmanson Medical Foundation Chia-Yi Christian Hospital, Chia-Yi 60002, Taiwan, R.O.C.

Received February 8, 2012; Accepted March 19, 2012

DOI: $10.3892 /$ or.2012.1789

\begin{abstract}
Gene therapy mediated by murine leukemia virus (MLV)-based replicating retrovirus vector (RRV) was previously proven to be highly effective in tumor cell killing, resulting in significant suppression of tumor growth in vivo. Recently, we developed a different form of RRV which is derived from another retrovirus, gibbon ape leukemia virus (GALV), as a cancer therapeutic agent. We compared the gene delivery efficiency and antitumor effects in the two types of RRV in experimental hepatocellular carcinoma (HCC). Our results show that both RRVs can efficiently spread throughout entire HCC cell populations in vitro and achieve high transduction efficiency in HCC xenografts in vivo, while GALV $\mathrm{RRV}$, in general, exhibited more rapid replication kinetics in the tumors. In vitro, substantial HCC cell killing was achieved even when initially only $1 \%$ of the HCC cells were producing RRVs that express the yeast cytosine deaminase suicide gene, indicating that the high efficiency of gene transfer by replicative spread of RRVs greatly increased suicide gene toxicity. In vivo, GALV RRV-mediated suicide gene therapy efficiently suppressed HCC tumor growth and no detectable RRV signals were observed in extratumoral tissues, showing promise in using GALV RRV as a cancer therapeutic agent.
\end{abstract}

\section{Introduction}

Gene therapy approaches have investigated oncolytic virus vectors that replicate specifically in tumor cells and subse-

Correspondence to: Dr Chien-Kuo Tai, Department of Life Science, National Chung Cheng University, 168 University Road, Min-Hsiung, Chia-Yi 62102, Taiwan, R.O.C.

E-mail: biockt@ccu.edu.tw

*Contributed equally

Key words: oncolytic virotherapy, retrovirus, cytosine deaminase, hepatocellular carcinoma quently kill these cells (1-3). Among the different oncolytic virus vectors being tested, the replicating retrovirus vector (RRV) based on the murine leukemia virus (MLV) exhibits some unique characteristics (4). MLV displays intrinsic tumor selectivity due to its inability to infect non-dividing cells (5-7), and can achieve highly efficient and stable gene transfer throughout entire solid tumors in vivo (8). While not intrinsically oncolytic, MLV can be armed with a suicide gene that will be delivered to tumor cells as the virus replicates $(9,10)$. As suicide genes encode prodrug-converting enzymes that catalyze intracellular production of toxic metabolites from a nontoxic prodrug, simultaneous killing of tumor cells can be triggered by prodrug administration.

In addition to MLV-based RRV, we have developed another version of RRV which is derived from the gibbon ape leukemia virus (GALV) (11). The GALV RRV retains a fully native viral genome and thus possesses the ability to replicate in cells. In addition to an intact GALV genome, the RRV contains an internal ribosome entry site (IRES)-GFP sequence between the env gene and the $3^{\prime}$ untranslated region (3' UTR). While the GALV RRV infects a target tumor cell, each infected cell itself becomes a virus-producing cell to sustain further infection events, thus greatly increasing gene delivery efficiency in vivo. To evaluate the potential of GALV RRV as a therapeutic agent for cancer treatment, we incorporated the yeast cytosine deaminase (CD) suicide gene into the RRV (12). Since the RRV can achieve efficient delivery of the CD gene that permanently integrates into the target cell genome, the CD enzyme would kill the cell through deamination of the prodrug 5-fluorocytosine (5-FC) to form the highly toxic 5-fluorouracil (5-FU) (13,14).

In the study, we aimed to explore the use of MLV-derived and GALV-derived RRVs for suicide gene therapy of hepatocellular carcinoma (HCC). We first compared the gene delivery efficiency of the two RRVs in HCC cells and tumor xenografts. Next, we examined the survival of transduced HCC cells as well as tumor growth after inoculation of RRVs followed by 5-FC treatment. We evaluated whether the higher transduction levels achieved by RRVs have the potential to enhance the efficacy of CD suicide gene therapy for HCC. 


\section{Materials and methods}

Construction of retroviral vectors. Plasmids pACE-GFP and pACE-CD are replication-competent amphotropic MLV vectors containing an IRES-GFP or IRES-CD cassette positioned between amphotropic env and $3^{\prime}$ UTR $(9,15)$. Plasmid pGS4-GFP is a replication-competent GALV vector containing an IRES-GFP positioned between the env gene and 3' UTR (11). The CD gene was amplified from pACE-CD by PCR and used to replace the GFP sequence in pGS4-GFP, generating plasmid pGS4-CD.

Viral vector production and infection. The transformed human embryonic kidney cell line 293T and the human hepatoma cell lines Huh-7, HepG2 and HA22T were cultivated in Dulbecco's modified Eagle's medium with $10 \%$ fetal bovine serum (Invitrogen, Carlsbad, CA, USA). Viral vectors were produced by transient transfection of the virus-encoding plasmids into 293T cells using calcium phosphate precipitation as previously described (16). Two days post-transfection, the virus-containing supernatant was collected, passed through $0.45-\mu \mathrm{m}$ syringe filters, and stocked at $-80^{\circ} \mathrm{C}$ for later use. For in vitro experiments, $4 \mu \mathrm{g} / \mathrm{ml}$ polybrene (Sigma, St. Louis, MO, USA) was added to the medium at the time of infection. For titer determination, after incubation of target cells with viral supernatant for $24 \mathrm{~h}, 25 \mu \mathrm{M}$ AZT (Sigma) was supplied to prevent ongoing virus replication as previously described (17), and the cells were analyzed for GFP expression using a FACScan (Becton-Dickinson, Franklin Lakes, NJ, USA). Viral titer was represented as transducing units (TU).

Cell viability analysis. Huh-7 and HepG2 cells transduced with ACE-GFP or ACE-CD were seeded onto replicate 96-well plates (2,000 cells/well). After overnight culture, the cells were exposed to $0.5 \mathrm{mM} 5-\mathrm{FC}$, and cell viability was determined daily by the MTS assay using the CellTiter Aqueous One Solution Cell Proliferation Assay kit (Promega, Madison, WI, USA). In a separate experiment, ACE-CD or GS4-CD infected Huh-7 cells were mixed with uninfected cells at a ratio of $1,10,50$ or $100 \%$ of the cell population and seeded onto 96-well plates. After overnight culture, the cells were exposed to $0.5 \mathrm{mM} 5$-FC, and viable cells were determined by the MTS assay.

Viral replication assay in vitro. HCC cells transduced with ACE-GFP or GS4-GFP were mixed with uninfected parental cells at a ratio of $5 \%$ of the cell population and seeded onto 6-well plates. At various times after mixing, the total cell population was analyzed for GFP expression by flow cytometry in order to estimate the spread of viral vectors. In a separate assay, HCC cells seeded on 6-well plates were infected with ACE-GFP or GS4-GFP at a multiplicity of infection (MOI) of 0.05 in the presence of $4 \mu \mathrm{g} / \mathrm{ml}$ polybrene. At various time points post infection, the cells were also analyzed for GFP expression by flow cytometry.

Viral replication assay in subcutaneous and orthotopic tumor models. For the subcutaneous tumor model, 5x10 ${ }^{6}$ Huh-7 cells were implanted into the right dorsal flank of athymic nude mice (National Laboratory Animal Center, Taiwan). When the tumor volumes reached $\sim 50-60 \mathrm{~mm}^{3}, 2.5 \times 10^{5}$ TU ACE-GFP or $1 \times 10^{5}$ TU GS4-GFP was injected into the center of each tumor. At various time points after viral vector inoculation, the tumors were excised, minced, and digested with $0.2 \%$ collagenase/dispase (Roche Diagnostics Corp., Indianapolis, IN, USA). The dissociated cells were spun down, resuspended in PBS, and passed through a $100-\mu \mathrm{m}$ cell strainer, and the cells were immediately subjected to flow cytometry for GFP expression analysis. For the orthotopic tumor model, nude mice were intrahepatically implanted with cell suspensions $\left(2 \times 10^{6}\right)$ consisting of GS4-GFP transduced Huh-7 mixing with uninfected Huh-7 at a ratio of $5 \%$ of the cell population. Four and seven weeks after tumor implantation, the mice were sacrificed and the tumor cells were harvested as previously described and analyzed by flow cytometry for GFP expression.

In vivo therapeutic effect of $G S 4-C D / 5 F C$. Five athymic nude mice were inoculated with $5 \times 10^{6}$ GS4-CD pretransduced Huh-7 cells and 15 mice were inoculated with $5 \times 10^{6}$ parental Huh-7 cells into the right dorsal flank. When the tumor volumes reached 50-60 $\mathrm{mm}^{3}, 1 \times 10^{5}$ TU GS4-CD $(\mathrm{n}=10)$ or PBS vehicle $(n=5)$ was injected into the center of each parental Huh-7 tumors. Eleven days after intratumoral injections of GS4-CD or PBS, all mice received intraperitoneal injections of 5-FC $(500 \mathrm{mg} / \mathrm{kg})$ or PBS, once every other day, for a total of 16 treatments. The tumor volumes were measured every 2-3 days.

Real-time PCR analyses of virus spread in animals. To detect any integrated GS4-CD sequences in genomes, real-time PCR was performed using an ABI Prism 7700 sequence detector. The primers were designed to target the GALV envelope (forward: 5'-CCTATTACTCCTCCTTCTGTTG-3'; reverse: 5'-GGGCCTGATATTTTTGTCTAAG-3'). In order to ascertain the precise amounts of input genomic DNA the apolipoprotein $\mathrm{B}$ gene was also quantified as an internal control (forward: 5'-CACGTGGGCTCCAGCATT-3'; reverse: 5'-TCACCAGTCATTTCTGCCTTTG-3'). Real-time PCR was performed in $25 \mu \mathrm{l}$ of reaction mixture containing $15 \mathrm{ng}$ of genomic DNA, $12.5 \mu \mathrm{l}$ of $2 \mathrm{X}$ SYBR-Green real-time PCR master mix (Toyobo, Osaka, Japan) and $300 \mathrm{nM}$ of each primer. Products were amplified by 45 cycles of successive incubation at $95^{\circ} \mathrm{C}$ for $15 \mathrm{sec}$ and at $60^{\circ} \mathrm{C}$ for $1 \mathrm{~min}$. A standard curve for GS4-CD copy number was generated by amplification of serially diluted pGS4-CD plasmid at specific copy numbers mixed into genomic DNA from spleen cells of uninfected mice.

\section{Results}

Cytotoxic effect of CD plus 5-FC in HCC cells. We have previously demonstrated that ACE-CD (Fig. 1) could efficiently inhibit the growth of human U87 glioma cells and murine CT26 colorectal cancer cells in vitro and in vivo after 5-FC administration $(9,18)$. To examine whether such therapeutic effects could also be achieved in HCC, Huh-7 and HepG2 cells were first transduced with ACE-GFP or ACE-CD. We exposed these cells to 5 -FC at the concentration of $0.5 \mathrm{mM}$, and the cell viability was determined by the MTS assay and normalized to that of the negative control. These results show that 


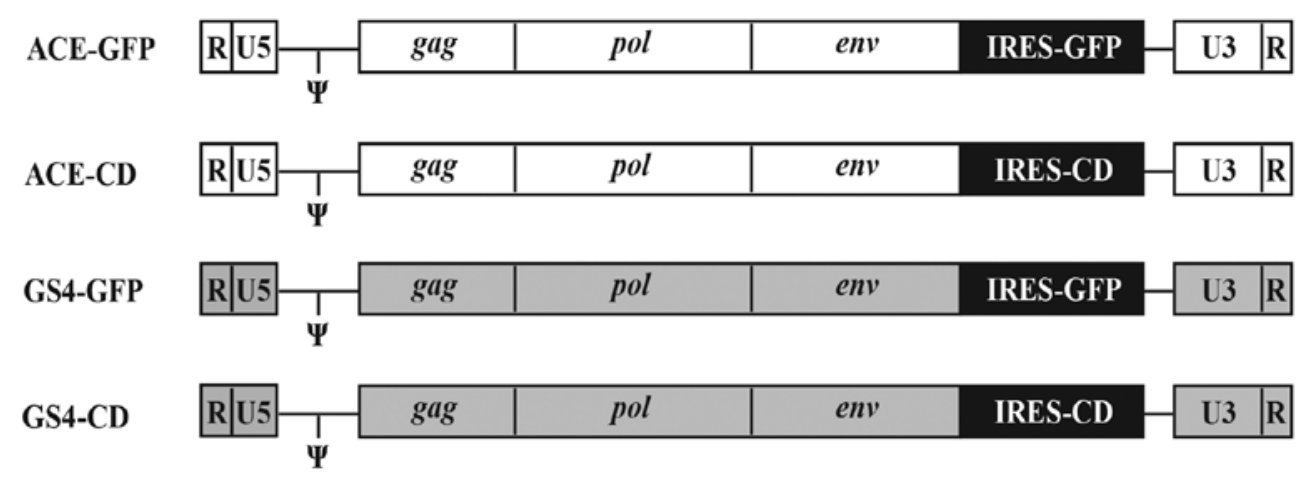

Figure 1. Schematic representation of replicating retrovirus vectors. ACE-GFP and ACE-CD are replication-competent amphotropic MLV vectors containing an IRES-transgene cassette positioned between amphotropic env and 3' UTR. GS4-GFP and GS4-CD are replication-competent GALV vectors containing an IRES-transgene cassette. Unshaded regions represent sequences derived from MLV and grey shaded regions represent those derived from GALV. IRES, internal ribosome entry site; $\Psi$, packaging signal.
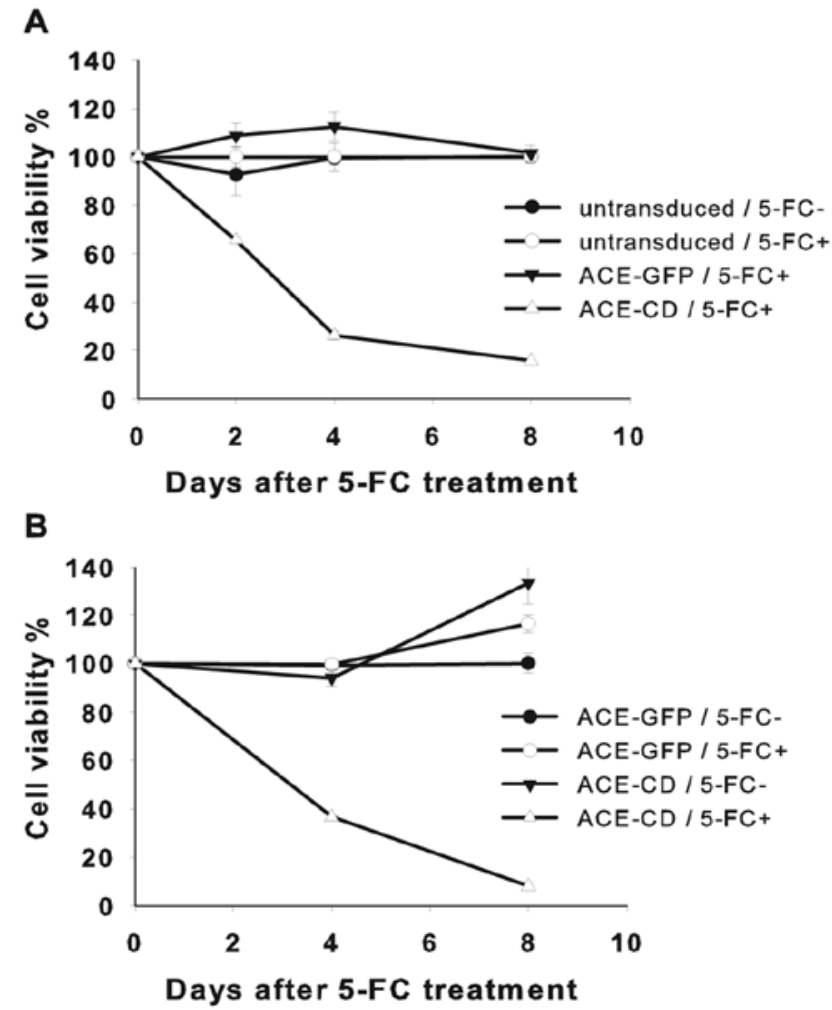

Figure 2. Cytotoxic effect of CD plus 5-FC in vitro. (A) RRV transduced and untransduced Huh-7 cells were exposed to $0.5 \mathrm{mM}$ 5-FC, and viability of cells was determined daily by the MTS assay. (B) RRV transduced HepG2 cells were exposed to $0.5 \mathrm{mM} 5$-FC, and viability of the cells was determined by the MTS assay on the indicated days. 5-FC-, without 5-FC incubation; 5-FC+, with 5-FC incubation.

ACE-CD transduction plus 5-FC treatment caused significant cytotoxicity to both HCC lines, indicating that the therapeutic strategy is applicable to treat HCC as well (Fig. 2).

RRVs efficiently replicate in HCC cells and achieve potent cell killing activity. To compare the replication kinetics of ACE-GFP and GS4-GFP in HCC, we prepared a cell mixture in which HCC cells infected with ACE-GFP or GS4-GFP were mixed with uninfected parental cells at a ratio of $5 \%$ of the cell population and were seeded onto 6-well plates. Flow cytometric analyses showed that both RRVs could efficiently spread throughout entire cell populations, while GS4-GFP exhibited more rapid replication kinetics in Huh-7 and HA22T (Fig. 3A). We further evaluated the efficacy of RRVs to transduce HCC in culture by infecting three HCC lines with ACE-GFP or GS4-GFP at an MOI of 0.05 and checked viral transduction by flow cytometry. Again, our results showed that both RRVs could efficiently transduce each HCC line, while GS4-GFP exhibited faster replication kinetics in Huh-7 cells (Fig. 3B). To determine if the higher transduction efficiency of the GS4 vector contributes to a better cytotoxic effect in HCC cells, GS4-CD or ACE-CD transduced Huh-7, mixed with uninfected Huh-7 at varying percentages, were treated with 5-FC for 3 and 6 days. When even as few as $1 \%$ of the Huh-7 cells in culture initially expressed $\mathrm{CD}$, substantial cell death was observed over time after incubation with 5-FC (Fig. 3C). The efficiency of cell killing was highly dependent on the initial fraction of cells in the culture expressing CD, as higher percentages of transduced cells in the culture achieved correspondingly faster clearance of the tumor cell population. The efficiency of cell killing was also positively correlated with the kinetics of viral replication, as a higher level of cytotoxicity was achieved by fast replicated GS4 vector.

Efficient and progressive spread of RRVs in subcutaneous and orthotopic HCC. To evaluate the ability of RRVs to achieve efficient transduction in solid tumors in vivo, we tested RRVs in a subcutaneous Huh-7 tumor model. A single dose of $2.5 \times 10^{5}$ TU ACE-GFP or $1 \times 10^{5}$ TU GS4-GFP was injected into a pre-established subcutaneous Huh-7 tumor model. The spread of RRVs in tumors was examined by quantification of GFP expression at various time points after viral vector injection. The results demonstrate that both RRVs are capable of efficient and progressive transduction of the Huh-7 solid tumors in vivo, while again GS4-GFP exhibited more rapid replication kinetics in the tumors (Fig. 4A). Furthermore, to study if GS4-GFP could efficiently spread in orthotopic liver tumors, we prepared a cell mixture in which Huh-7 cells infected with GS4-GFP were mixed with uninfected Huh-7 cells at a ratio of $5 \%$ of the cell population and was implanted to livers of nude mice. Through flow cytometric analysis of dissected orthotopic tumors, the percentage of GFP-positive 

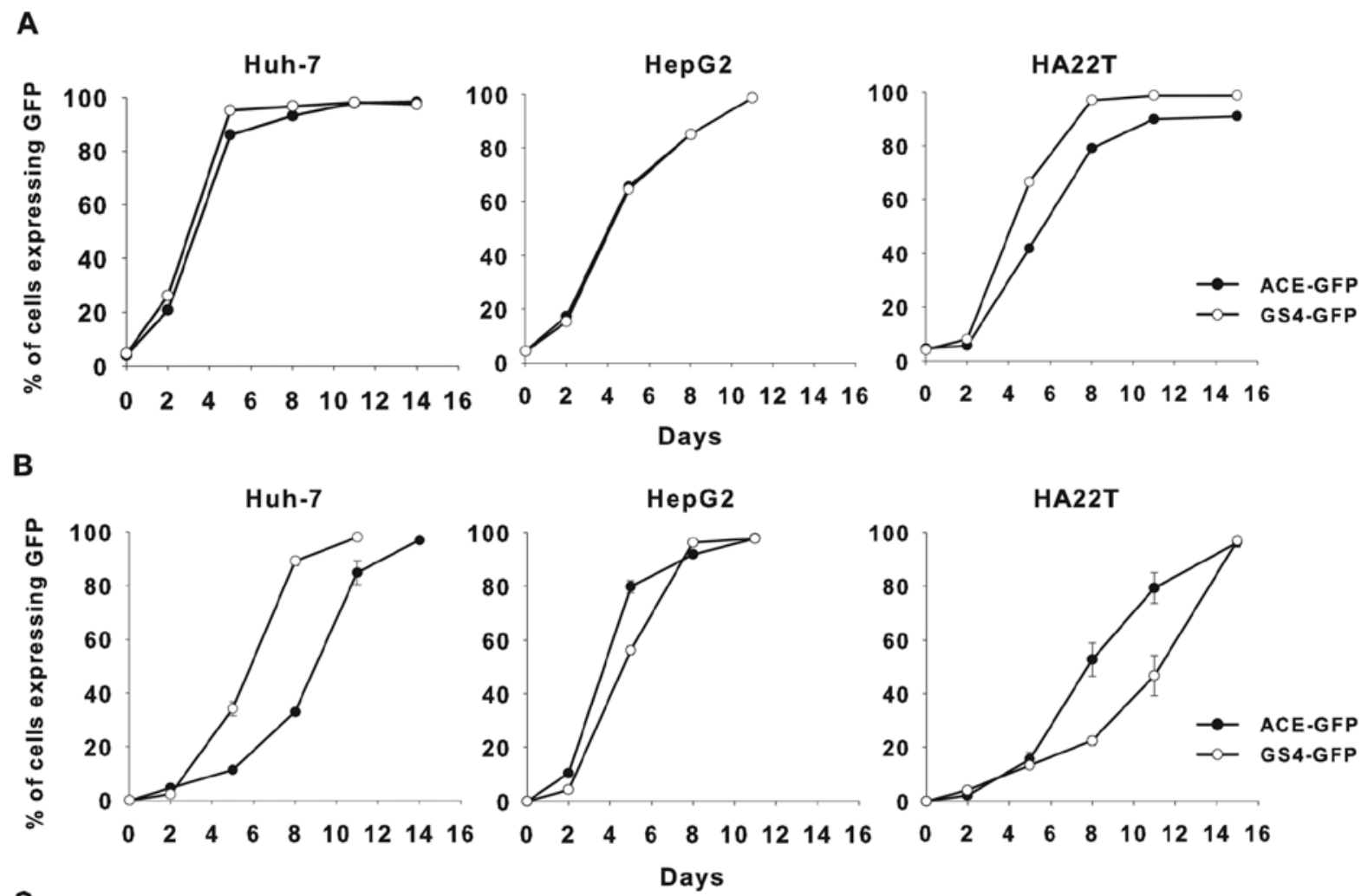

C

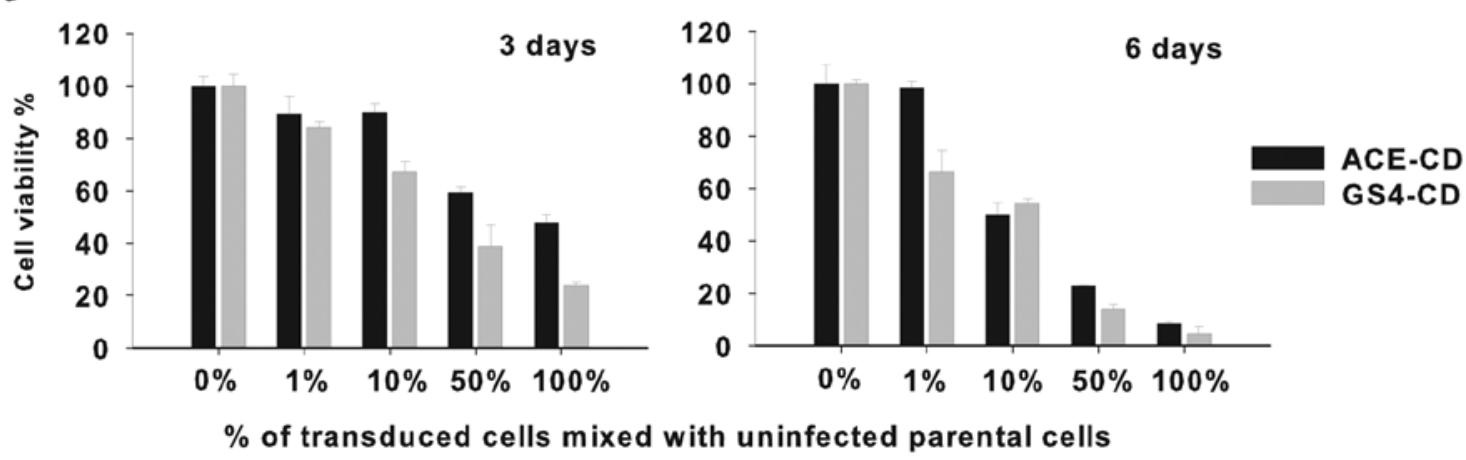

Figure 3. Replication kinetics and therapeutic effect of RRVs in vitro. (A) ACE-GFP-transduced or GS4-GFP-transduced human HCC cells were mixed with uninfected $\mathrm{HCC}$ cells at a ratio of 5\% of the cell population and seeded onto culture plates. Various times after mixing, the total cell population was analyzed for GFP expression by flow cytometry. (B) Human HCC cells Huh-7, HepG2, and HA22T were inoculated with ACE-GFP or GS4-GFP at an MOI of 0.05. At various time points after inoculation, the cells were analyzed for GFP expression by flow cytometry. (C) ACE-CD or GS4-CD transduced Huh-7 cells and uninfected cells were mixed at various initial ratios $(0,1,10,50$, and $100 \%$ of transduced cells) and seeded onto 96 -well plates. The mixed cell populations were exposed to $0.5 \mathrm{mM} 5$-FC, and viability of cells was determined by MTS assay on the indicated days. Shown values of viability percentage of the cells were normalized to that of the negative control.

Huh-7 cells was determined to be 74.5 and $80.6 \%$ at 4 and 7 weeks post tumor implantation, demonstrating that the GS4 vector was capable of efficient replication and gene transduction in orthotopic tumors (Fig. 4B).

Therapeutic effect of GS4-CD in vivo. To determine whether the high level of transduction efficiency achieved by the GS4 vector could lead to therapeutic benefit in vivo, we injected $1 \times 10^{5} \mathrm{TU}$ GS4-CD or PBS vehicle into a naïve pre-established subcutaneous Huh-7 tumor model. Eleven days after intratumoral injections of GS4-CD or PBS, the mice received intraperitoneal injections of 5-FC or PBS every other day. Mice bearing GS4-CD pretransduced Huh-7 subcutaneous tumors were treated with $5-\mathrm{FC}$ as a positive control. The data reveal that the GS4-CD/5-FC-treated group demonstrated significant suppression of tumor growth, compared with the two control groups (PBS/PBS and GS4-CD/PBS) (Fig. 5).

Distribution of $R R V$ during progressive spread in vivo. To estimate the safety of RRV for gene therapy of HCC, we analyzed the genomic DNA extracted from tumors and extratumoral organs of GS4-inoculated mice by real-time quantitative PCR using primers specific for the GALV envelope gene. As expected, RRV could be readily detected in the GS4 transduced Huh-7 tumors by PCR amplification of the GALV envelope sequence (data not shown). However, no detectable GS4 signal was observed in genomic DNA from extratumoral organs including liver, spleen, intestine, heart and lung in the same animals. 
A

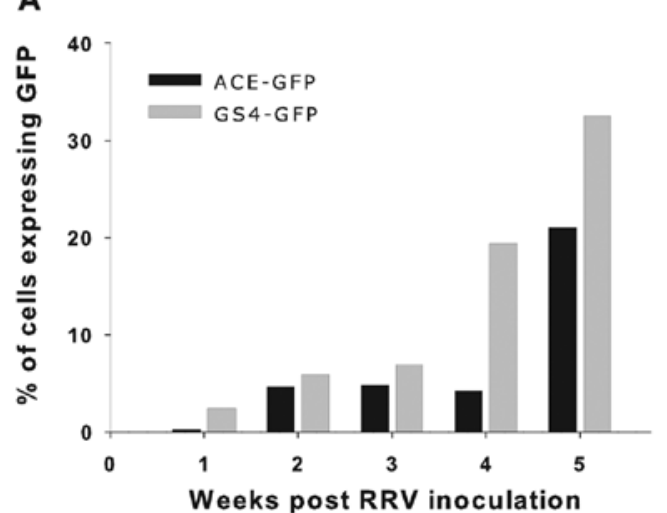

B

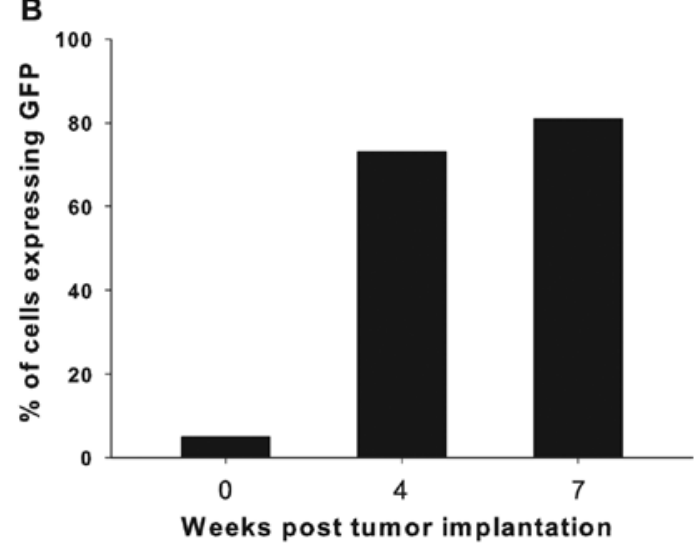

Figure 4. Replicative spread of RRVs in solid tumors. (A) Spread of ACE-GFP and GS4-GFP in subcutaneous HCC. ACE-GFP $\left(2.5 \times 10^{5} \mathrm{TU}\right)$ or GS4-GFP $\left(1 \times 10^{5} \mathrm{TU}\right)$ was injected into a pre-established subcutaneous Huh-7 tumor model in nude mice. The spread of RRVs in tumors was examined by quantification of GFP expression at various time points after viral vector injection. (B) Spread of GS4-GFP in orthotopic HCC. A cell suspension consisting of GS4-GFP transduced Huh-7 cells mixed with uninfected cells at a ratio of $5 \%$ of the cell population was implanted to the liver of nude mice. Through flow cytometric analysis of dissected tumors, the percentage of GFP-positive cells was determined to be 74 and $81 \%$ at 4 and 7 weeks, respectively, after implantation.

\section{Discussion}

Although traditional replication-defective retrovirus vectors have been applied for a number of clinical cancer trials, the low efficiency of gene delivery to tumors exhibited by the vectors becomes their major limitation for gene therapy of cancer (19-21). In the present study, we demonstrate that RRVs can efficiently replicate in human HCC cells in vitro, and can achieve efficacious levels of gene transfer in subcutaneous and orthotopic Huh-7 tumors in vivo. Cell viability assays showed significant cell killing by RRV-CD even with an initial inoculum as low as $1 \%$ of the target cell population, indicating that the high efficiency of gene transfer by progressive replicative spread of the vectors greatly increased suicide gene toxicity. Real-time PCR analyses demonstrated that, after intratumoral administration of RRV in vivo, RRV replication was limited to the tumor tissue itself, suggesting that the absolute requirement for cell division results in a high degree of tumor selectivity. Furthermore, higher levels of tumor selectivity of RRV may be achieved by replacement of the retroviral promoter with tissue-specific transcriptional elements to target viral gene

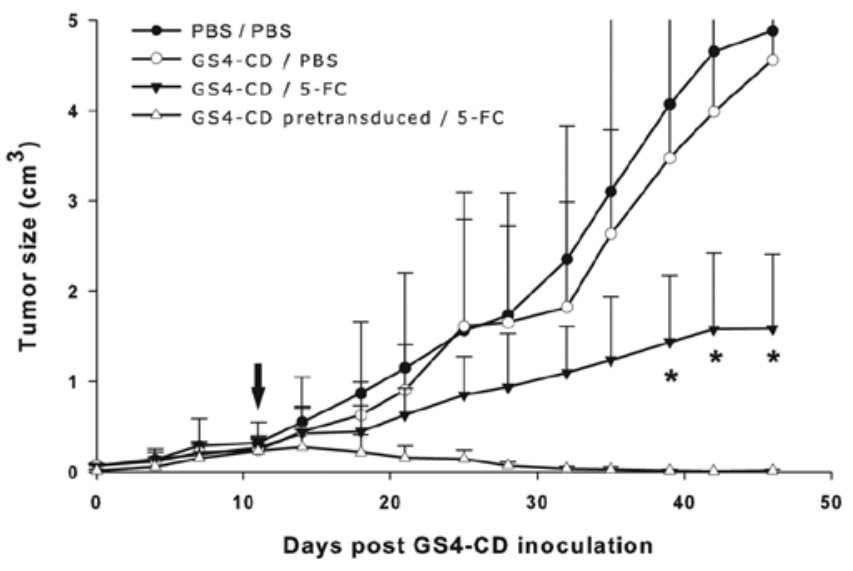

Figure 5. Therapeutic effect achieved by RRV gene therapy. GS4-CD (1x10 TU) or PBS vehicle was injected into a pre-established subcutaneous Huh-7 tumor model in nude mice. Eleven days after intratumoral injections of GS4-CD or PBS, the mice received intraperitoneal injections of 5-FC or PBS every other day, for a total of 16 treatments. Mice bearing GS4-CD pretransduced Huh-7 subcutaneous tumors were treated with 5-FC as a positive control. Statistically significant differences in tumor size between the GS4$\mathrm{CD} / 5$-FC treated group and the 2 control groups PBS/PBS and GS4-CD/PBS were observed. ${ }^{*} \mathrm{P}<0.05$. Solid arrow, beginning of 5 -FC treatments.

expression to particular tissues, thus limiting virus replication to tumors (22).

Transductions of three HCC cell lines with ACE-GFP and GS4-GFP at a low MOI showed that both the ACE and the GS4 vectors could spread throughout the entire cell populations, while the GS4 vector, in general, exhibited more rapid replication kinetics in HCC cells. The efficiency of cell killing was also correlated with the kinetics of RRV replication, as the higher spread efficiency of GS4-CD achieved a higher level of cytotoxicity after 5-FC administration. Since ACE and GS4 vectors utilize different cellular receptors (Pit-2 and Pit-1 phosphate transporters, respectively) for viral entry (23), co-infection of HCC cells with ACE and GS4 vectors supplied with different suicide genes may be employed to achieve synergistic cytotoxic effects, thus greatly augmenting gene therapy efficacy $(11,24)$.

In vivo viral spread assays also demonstrated that the GS4 vector can stably propagate in subcutaneous Huh-7 tumors, achieving relatively higher transduction efficiencies compared to the ACE vector. Suicide gene transfer by GS4-CD followed by 5 -FC administration resulted in a significant tumor inhibitory effect on Huh-7 xenografts, although tumors were not completely suppressed. It is likely that these animals were treated with 5-FC while their tumors were still only partially transduced with GS4-CD; therefore, any untransduced tumor that escaped cytotoxic killing presumably resumed growth. This suggests that optimally effective doses of the RRV may not have been delivered to achieve a sufficient level of transduction throughout the tumor mass before initiating 5-FC administration. Therefore, a higher or repeat dose of RRV might achieve even more efficient tumor inhibition in the Huh-7 tumor model. In addition, continuous prodrug administration may further suppress growth of tumors due to the long-term persistence and stable expression of the RRV (10). Such degree of viral persistence is unique among oncolytic viruses and may be especially advantageous in treating highly 
metastatic tumors, as any infected tumor cell will be converted into a constant source of ongoing virus production that will spread the vector to ectopic sites as those cells metastasize. In summary, the use of RRV may represent a promising therapeutic strategy for HCC because of its ability to efficiently and selectively transduce tumor cells in vivo, achieving stable integration and persistent transgene expression concomitant with progressive virus replication within the tumor.

\section{Acknowledgements}

This study was supported by the Ditmanson Medical Foundation Chia-Yi Christian Hospital and grant NSC992320-B-194-004-MY3 from the National Science Council, Taiwan.

\section{References}

1. Smerdou C, Menne S, Hernandez-Alcoceba R and GonzalezAseguinolaza G: Gene therapy for $\mathrm{HCV} / \mathrm{HBV}$-induced hepatocellular carcinoma. Curr Opin Investig Drugs 11: 1368-1377, 2010

2. Fukazawa T, Matsuoka J, Yamatsuji T, Maeda Y, Durbin ML and Naomoto Y: Adenovirus-mediated cancer gene therapy and virotherapy (Review). Int J Mol Med 25: 3-10, 2010.

3. Kirn D, Martuza RL and Zwiebel J: Replication-selective virotherapy for cancer: biological principles, risk management and future directions. Nat Med 7: 781-787, 2001.

4. Tai CK and Kasahara N: Replication-competent retrovirus vectors for cancer gene therapy. Front Biosci 13: 3083-3095, 2008.

5. Lewis PF and Emerman M: Passage through mitosis is required for oncoretroviruses but not for the human immunodeficiency virus. J Virol 68: 510-516, 1994.

6. Miller DG, Adam MA and Miller AD: Gene transfer by retrovirus vectors occurs only in cells that are actively replicating at the time of infection. Mol Cell Biol 10: 4239-4242, 1990

7. Varmus HE, Padgett T, Heasley S, Simon G and Bishop JM: Cellular functions are required for the synthesis and integration of avian sarcoma virus-specific DNA. Cell 11: 307-319, 1977.

8. Wang WJ, Tai CK, Kasahara N and Chen TC: Highly efficient and tumor-restricted gene transfer to malignant gliomas by replication-competent retroviral vectors. Hum Gene Ther 14: 117-127, 2003.

9. Tai CK, Wang WJ, Chen TC and Kasahara N: Single-shot, multicycle suicide gene therapy by replication-competent retrovirus vectors achieves long-term survival benefit in experimental glioma. Mol Ther 12: 842-851, 2005.
10. Tai CK, Wang W, Lai YH, et al: Enhanced efficiency of prodrug activation therapy by tumor-selective replicating retrovirus vectors armed with the Escherichia coli purine nucleoside phosphorylase gene. Cancer Gene Ther 17: 614-623, 2010.

11. Lu YC, Luo YP, Wang YW and Tai CK: Highly efficient gene transfer to solid tumors in vivo by tumor-selective replicating retrovirus vectors. Int J Mol Med 25: 769-775, 2010.

12. Kievit E, Bershad E, Ng E, et al: Superiority of yeast over bacterial cytosine deaminase for enzyme/prodrug gene therapy in colon cancer xenografts. Cancer Res 59: 1417-1421, 1999.

13. Kievit E, Nyati MK, Ng E, et al: Yeast cytosine deaminase improves radiosensitization and bystander effect by 5 -fluorocytosine of human colorectal cancer xenografts. Cancer Res 60: 6649-6655, 2000.

14. Nakamura H, Mullen JT, Chandrasekhar S, Pawlik TM, Yoon SS and Tanabe KK: Multimodality therapy with a replicationconditional herpes simplex virus 1 mutant that expresses yeast cytosine deaminase for intratumoral conversion of 5-fluorocytosine to 5-fluorouracil. Cancer Res 61: 5447-5452, 2001.

15. Logg CR, Logg A, Matusik RJ, Bochner BH and Kasahara N: Tissue-specific transcriptional targeting of a replication-competent retroviral vector. J Virol 76: 12783-12791, 2002.

16. Pear WS, Nolan GP, Scott ML and Baltimore D: Production of high-titer helper-free retroviruses by transient transfection. Proc Natl Acad Sci USA 90: 8392-8396, 1993.

17. Tai CK, Logg CR, Park JM, Anderson WF, Press MF and Kasahara N: Antibody-mediated targeting of replication-competent retroviral vectors. Hum Gene Ther 14: 789-802, 2003.

18. Hiraoka K, Kimura T, Logg CR, et al: Therapeutic efficacy of replication-competent retrovirus vector-mediated suicide gene therapy in a multifocal colorectal cancer metastasis model. Cancer Res 67: 5345-5353, 2007.

19. Andreadis ST, Roth CM, Le Doux JM, Morgan JR and Yarmush ML: Large-scale processing of recombinant retroviruses for gene therapy. Biotechnol Prog 15: 1-11, 1999.

20. Gordon EM and Hall FL: The 'timely' development of Rexin-G: First targeted injectable gene vector (Review). Int J Oncol 35: 229-238, 2009.

21. Rainov NG: A phase III clinical evaluation of herpes simplex virus type 1 thymidine kinase and ganciclovir gene therapy as an adjuvant to surgical resection and radiation in adults with previously untreated glioblastoma multiforme. Hum Gene Ther 11: 2389-2401, 2000.

22. Metzl C, Mischek D, Salmons B, Gunzburg WH, Renner M and Portsmouth D: Tissue- and tumor-specific targeting of murine leukemia virus-based replication-competent retroviral vectors. J Virol 80: 7070-7078, 2006.

23. Overbaugh J, Miller AD and Eiden MV: Receptors and entry cofactors for retroviruses include single and multiple transmembrane-spanning proteins as well as newly described glycophosphatidylinositol-anchored and secreted proteins. Microbiol Mol Biol Rev 65: 371-389, 2001.

24. Yue QH, Hu XB, Yin Y, et al: Inhibition of prostate cancer by suicide gene targeting the FCY1 and HSV-TK genes. Oncol Rep 22: 1341-1347, 2009. 